\title{
Genomic analysis on broiler-associated Clostridium perfringens strains and exploratory caecal microbiome investigation reveals key factors linked to poultry necrotic enteritis
}

\author{
Raymond Kiu', Joseph Brown², Harley Bedwell', Charlotte Leclaire', Shabhonam Caim', Derek Pickard³, \\ Gordon Dougan ${ }^{3}$, Ronald A. Dixon ${ }^{2}$ and Lindsay J. Hall ${ }^{1 *}$ (D)
}

\begin{abstract}
Background: Clostridium perfringens is a key pathogen in poultry-associated necrotic enteritis (NE). To date there are limited Whole Genome Sequencing based studies describing broiler-associated C. perfringens in healthy and diseased birds. Moreover, changes in the caecal microbiome during NE is currently not well characterised. Thus, the aim of this present study was to investigate C. perfringens virulence factors linked to health and diseased chickens, including identifying putative caecal microbiota signatures associated with NE.

Results: We analysed 88 broiler chicken C. perfringens genomes (representing 66 publicly available genomes and 22 newly sequenced genomes) using different phylogenomics approaches and identified a potential hypervirulent and globally-distributed clone spanning 20-year time-frame (1993-2013). These isolates harbored a greater number of virulence genes (including toxin and collagen adhesin genes) when compared to other isolates. Further genomic analysis indicated exclusive and overabundant presence of important NE-linked toxin genes including netB and tpeL in NE-associated broiler isolates. Secondary virulence genes including pfoA, $c p b 2$, and collagen adhesin genes $c n a$, $\mathrm{CnaA}$ and $\mathrm{cnaD}$ were also enriched in the NE-linked C. perfringens genomes. Moreover, an environmental isolate obtained from farm animal feeds was found to encode netB, suggesting potential reservoirs of NetB-positive $C$. perfringens strains (toxinotype G). We also analysed caecal samples from a small sub-set of 11 diseased and healthy broilers for exploratory microbiome investigation using $16 \mathrm{~S}$ rRNA amplicon sequencing, which indicated a significant and positive correlation in genus Clostridium within the wider microbiota of those broilers diagnosed with $\mathrm{NE}$, alongside reductions in beneficial microbiota members.

Conclusions: These data indicate a positive association of virulence genes including netB, pfoA, cpb2, tpel and cna variants linked to NE-linked isolates. Potential global dissemination of specific hypervirulent lineage, coupled with distinctive microbiome profiles, highlights the need for further investigations, which will require a large worldwide sample collection from healthy and NE-associated birds.
\end{abstract}

Keywords: Clostridium perfringens, Toxin, Phylogenomics, Necrotic enteritis, Poultry, Caecal microbiome, 16S rRNA analysis

\footnotetext{
* Correspondence: Lindsay.Hall@quadram.ac.uk

${ }^{1}$ Gut Microbes and Health, Quadram Institute Bioscience, Norwich, UK

Full list of author information is available at the end of the article
}

(c) The Author(s). 2019 Open Access This article is distributed under the terms of the Creative Commons Attribution 4.0 International License (http://creativecommons.org/licenses/by/4.0/), which permits unrestricted use, distribution, and reproduction in any medium, provided you give appropriate credit to the original author(s) and the source, provide a link to the Creative Commons license, and indicate if changes were made. The Creative Commons Public Domain Dedication waiver (http://creativecommons.org/publicdomain/zero/1.0/) applies to the data made available in this article, unless otherwise stated. 


\section{Background}

Broiler chickens are solely bred for meat production, and represent a key global livestock asset; with an estimated annual production of 50 billion birds worldwide [1]. As broilers reach slaughter weight at a young age (4-7 weeks) they are susceptible to several welfare and infection issues. Importantly, poultry Necrotic Enteritis $(\mathrm{NE})$, an inflammatory gut infection in chickens, is responsible for a loss of US\$6 billion per annum in the poultry industry, with $C$. perfringens reported to be the main causative agent [2-5].

NE-associated pathologies are mainly characterised by gaseous lesions and mucosa necrosis in gas-filled distended small intestines [6]. Proposed key C. perfringensassociated factors linked to NE include $\alpha$-toxin, and more recently NetB and TpeL (both pore-forming toxins) [7]. Other aetiological factors that have been shown to increase risk of NE include high-protein diets and environmental stressors, which may alter gut-associated microbial communities (i.e. the microbiota), host immunity, and coinfection with the poultry parasite Eimeria [8-10]. In addition, sub-clinical NE (SNE), which is a mild form of $\mathrm{NE}$, is represented by poor growth and small intestinal ulcerative lesions and has also been associated with C. perfringens colonisation [7, 11].

C. perfringens, a ultra-rapid-growing anaerobic Gram positive pathogen, is known to harbour an arsenal of > 20 toxins and has been associated with a wide range of gut diseases in animals, including poultry NE [5]. Specifically, toxin NetB is considered to be an essential C. perfringens virulence factor in NE pathogenesis, as determined in animal studies [2]. Expression of this pore-forming toxin has previously been reported to be higher (92\%) in NE chicken C. perfringens isolates, as compared to healthy chickens (29\%), thus supporting its role in disease progression [12]. This toxin is known to be encoded exclusively on conjugative plasmids, indicating horizontal gene transfer may play a role in dissemination to NetB-negative strains [13]. Collagen adhesin (encoded by gene cna and its variants) is another candidate disease determinant, which has been associated with chicken NE isolates in a recent bacterial genomic study [14].

The caecum represents the primary site for C. perfringens colonisation, which also contains the highest density of the chicken gut microbiota, therefore NE-induced alterations of this GI site are likely to reflect disease changes [15]. Moreover, the chicken caecal microbiome is known to play a protective role in pathogen resistance to other enteric pathogens, including Campylobacter jejuni, and as such intestinal microbiota disruption may impact development of $C$. perfringens-associated NE in broiler chickens, although direct biological impact is yet to be confirmed $[9,16,17]$.
At present there are only two smaller scale Whole Genome Sequencing (WGS) based studies on broilerassociated C. perfringens $[14,18]$. Thus, to further our understanding on C. perfringens dissemination and virulence profiles in the context of broiler-NE, we performed phylogenetics and in-depth comparative genomics on 88 chicken-associated C. perfringens isolates, (strains from public genome databases, alongside 22 newly isolated and sequenced strains). Moreover, it is unclear if and how the chicken caecal microbiome changes during NE development, therefore a small-scale microbiota profiling study was carried out to understand if there are any diseased-specific disturbances induced after C. perfringens infection.

\section{Results}

Phylogenetic analysis reveals a potentially important intercontinental lineage

We investigated 88 broiler-associated C. perfringens genomes (including 62 from NE-linked birds, 20 from healthy birds and 6 environmental isolates from broiler farms), spanning a 23-year period from 1993 to 2016 from 8 countries across European, Australasian and North American continents (Additional file 1: Table S1). Twenty-two C. perfringens genomes were sequenced and assembled specific to this study and the remaining isolates were publicly available. Broiler isolates Del1 and LLY_N11 were publicly available complete genomes sequenced using long-read sequencing and were included in analyses $[4,19]$. A Maximum Likelihood (ML) phylogenetic tree was assembled using 88 isolates from 20,194 SNPs identified from the alignment of 1810 core genes (Fig. 1). Clustering was assigned to define population structure via hierBAPS analysis; with the 88 isolates clustering into 5 major lineages.

The core gene alignment of 20,194 SNPs was subjected to SNP distance analysis. Phylogenetic clustering and pairwise SNP analysis suggested several sub-lineages that comprised multiple highly-similar strains including lineages IVa, IVb, Va, Vc, Vd and Vf (Fig. 1). Importantly, lineage $\mathrm{Vf}$ that consisted of 14 broiler isolates obtained from 4 different countries namely Australia, Canada, Denmark and USA (in between 1993 and 2013) displayed evident clonality; $65.0 \pm 52.8$ SNPs between strains, which was in contrast to the closest sub-lineage Ve, with pairwise SNP distance of $1474.0 \pm 162.6$ SNPs $(P<0.0001$; Fig. 2a-c). Average Nucleotide Identity (ANI) analysis also supported the apparent clonality in sub-lineage Vf $(n=14)$, with isolates demonstrating a pair-wise mean genome-wide ANI of $99.81 \pm 0.08 \%$, compared to closest sub-lineage pairwise ANI of $98.77 \pm$ $1.04 \%(P<0.0001$; Fig. 2 d). Delving further into this interesting cluster of multi-continental isolates, sublineage Vf3 displayed the highest genetic similarity of 


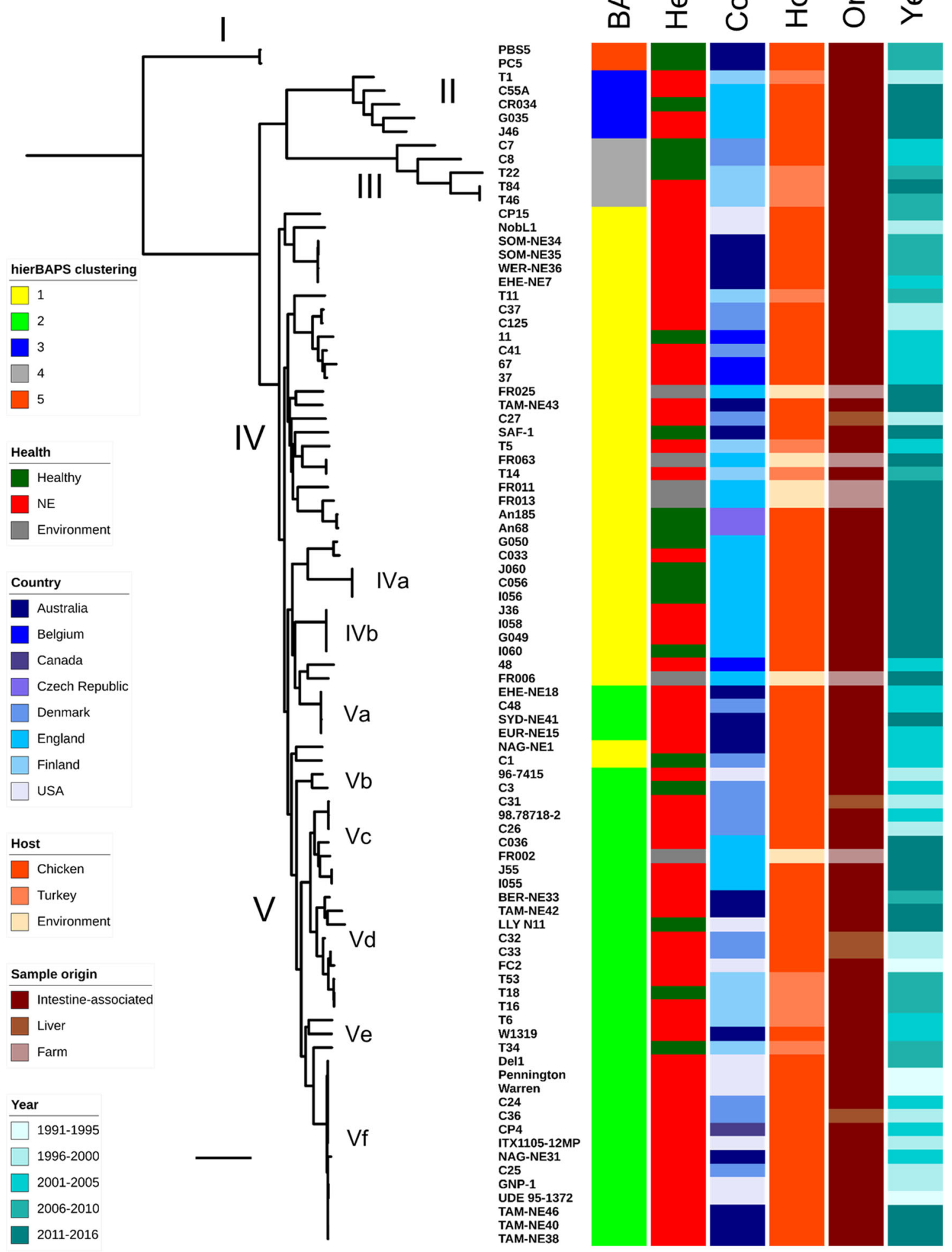

Fig. 1 Mid-point rooted maximum-likelihood tree based on 20,194 SNPs in 1810 core genes labelled by hierarchical Bayesian clusters (1-5), health states of hosts, country of isolates, poultry host species, sample origin and year of isolation. Scale bar, $\sim 2000$ SNPS 


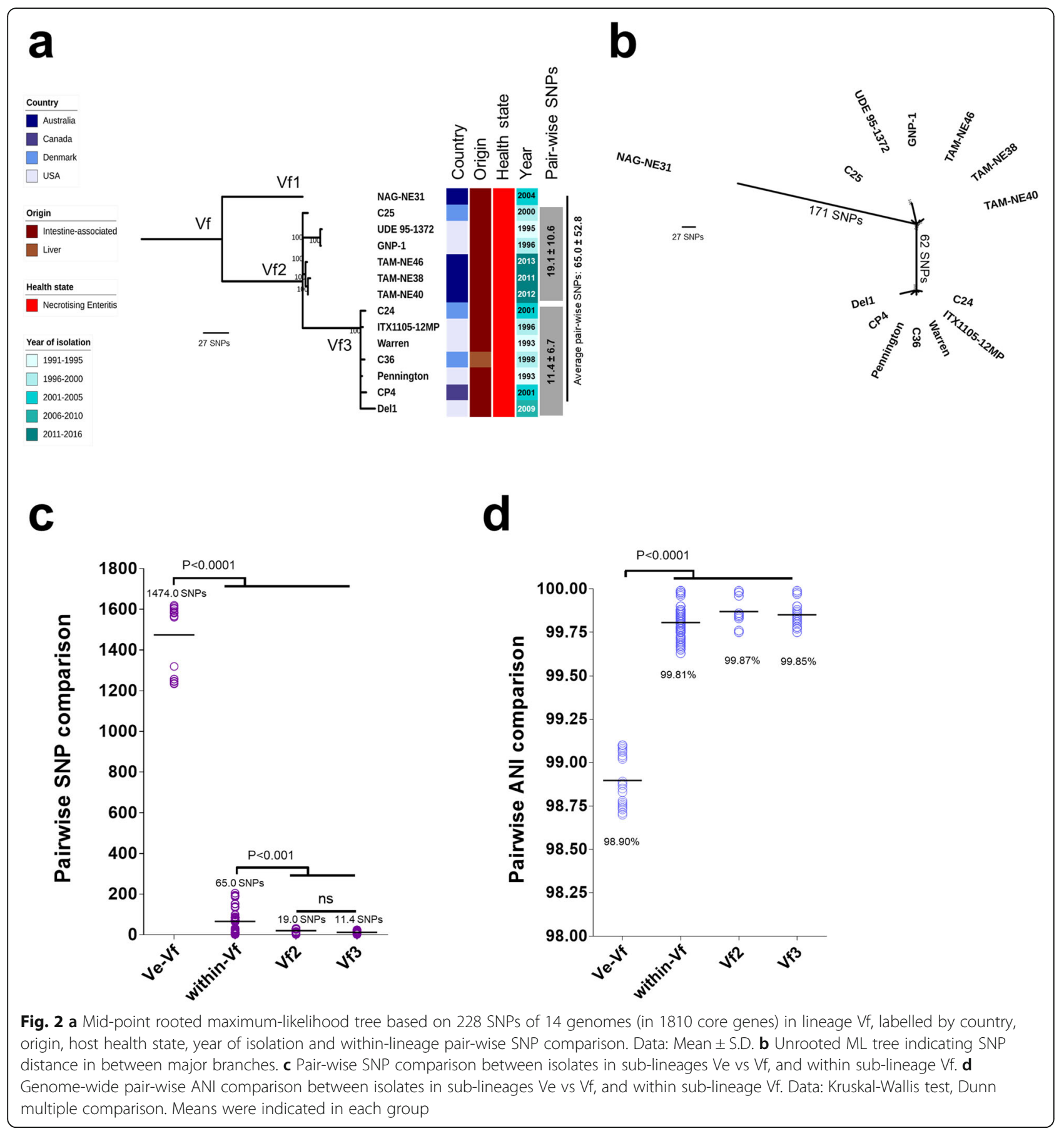

pairwise SNP distance of $11.4 \pm 6.7$ SNPs;7 isolates originated from Denmark, USA and Canada, spanning a period of 16 years (Fig. 2a). Sub-lineage Vf2 displayed a similar trend of low overall pairwise SNP distance of $19.1 \pm 10.6$ SNPs with these 6 isolates sourced from Australia, USA and Denmark. Australian isolate NAGNE31 was shown to be distant at 171 SNPs (Fig. 2b). This analysis suggests a potential widespread reservoir of this $C$. perfringens lineage given they are genetically highly-similar, and previous studies have indicated this species has a highly divergent genome [20-22].

Lineages IVa and IVb exclusively comprised newlysequenced $C$. perfringens isolates from English farms. Lineage IVb encompassed 4 isolates from 4 individual birds (J36, I060, G049 and I058), which were identical at strain level (0 SNPs), revealing potential intertransmission of $C$. perfringens strains among poultry farms in the same region. Interestingly, in lineage Va, 
Danish isolate C48 was shown to be highly similar to Australian isolates EHE-NE18 and EUR-NE15 at 7 SNPs difference, with all these isolates obtained in the same year 2002 (Additional file 2: Figure S2). Isolates in lineages Vd and Vc exhibited geographical similarity by country at minimal SNP counts.

\section{Virulence association analysis supports a hypervirulent $C$.}

\section{perfringens lineage}

C. perfringens encodes an arsenal of virulence-related genes including toxin, antimicrobial resistance (AMR), and collagen adhesin genes, which are linked with gut colonisation and pathogenesis [5]. Virulence plasmids are known to encode for NE-associated toxin genes netB and tpeL. We therefore carried out a comprehensive search on all the known virulence genes, AMR determinants and virulence plasmids encoded in each C. perfringens genome using both assembly-based approaches (as most public genomes were only available in assemblies) and read-mapping methods for plasmid searches (if sequencing reads available) (Fig. 3).

Initial analysis indicated that isolates in intercontinental lineage Vf consistently encoded more virulence genes, including netB and tpeL, thus a further comparative virulence gene analysis was performed (Additional file 2: Figure S3). Isolates within lineage Vf

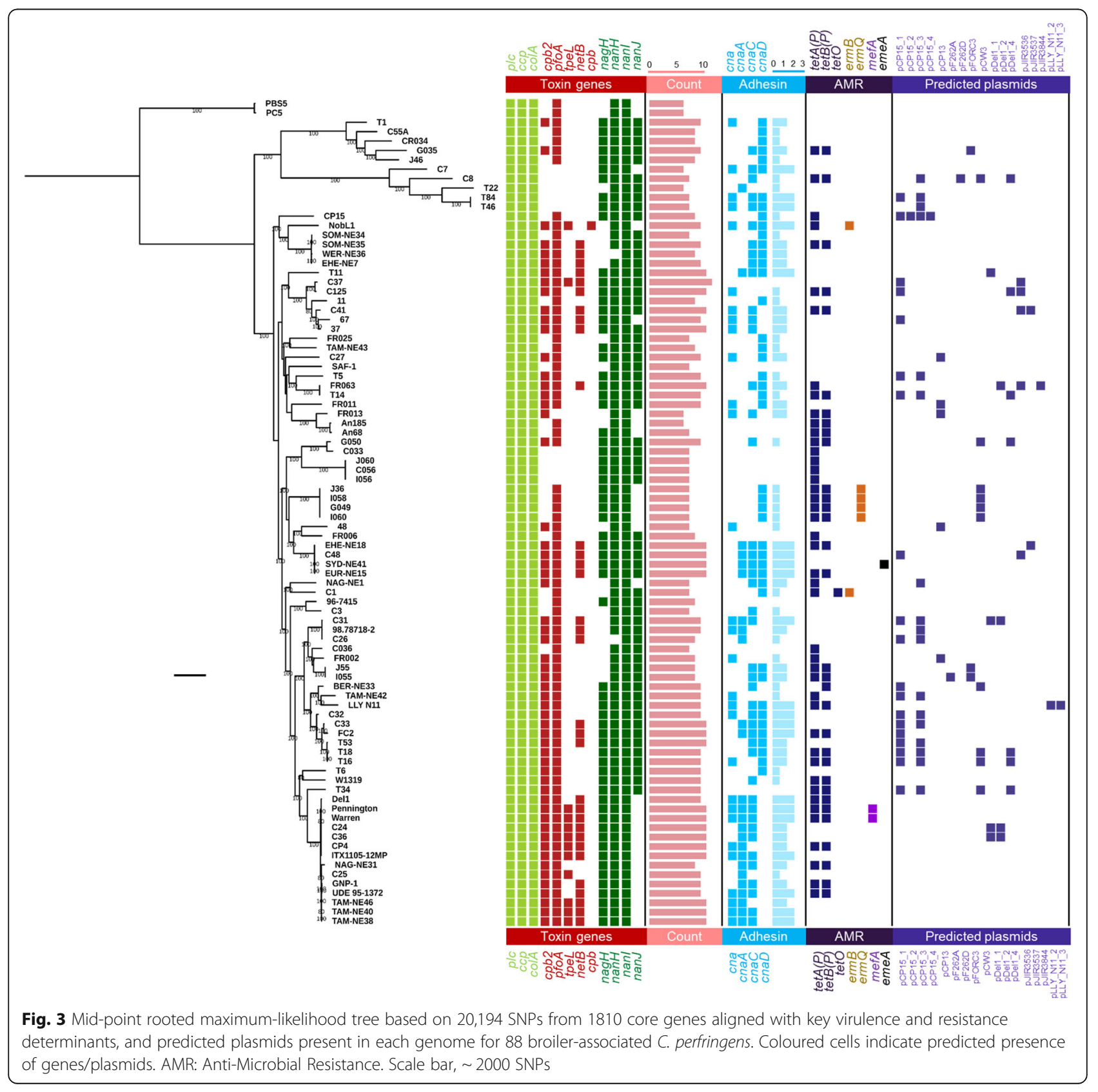


encoded significantly more toxin genes $(9.5 \pm 0.6$ toxin genes vs $8.2 \pm 1.3$ toxin genes; $P<0.0001)$ and collagen adhesin genes $(2.4 \pm 0.6$ vs $1.4 \pm 1.0, P=0.0005)$ when compared to the remaining isolates, suggesting this is potentially a 'hypervirulent' sub-lineage. This was supported by further analysis comparing virulence gene counts between sub-lineage $\mathrm{Vf}$, and remaining NE-linked isolates (Additional file 2: Figure S3); Vf isolates encoded more toxin $(9.5 \pm 0.6$ vs $8.7 \pm 1.1)$ and collagen adhesin genes $(2.4 \pm 0.6$ vs $1.7 \pm 0.9)$, when compared to the remaining $\mathrm{NE}$-associated $C$. perfringens strains.

Comparative analysis was also performed to define differences between NE-linked $(n=62)$ isolates and healthy-broiler isolates $(n=20$; Additional file 2: Figure S4). Both toxin genes $(8.8 \pm 1.1$ vs $7.2 \pm 1.0)$ and collagen adhesin genes $(1.9 \pm 0.9$ vs $0.8 \pm 1.0)$ were significantly elevated in NE-related isolates $(P<0.0001)$. Considering overall virulence (toxin + adhesin genes), NE-linked isolates encoded significantly more virulence genes $(10.8 \pm$ 1.7) than healthy-broiler isolates $(8.0 \pm 1.6 ; P<0.0001$; Fig. 4). Notably, isolates in lineage $\mathrm{Vf}$ encode the most virulence genes (12.0 \pm 1.0 genes).

To explore potential enrichment and correlation of NE-related toxin genes including netB, tpeL and other secondary toxin genes $p f o A$ and $c p b 2$, an association statistical analysis (Chi-square test) was performed (Additional file 2: Figure S5). Toxin gene netB was exclusively encoded in NE-linked isolates $(31 / 62 ; 50 \%)$ compared to healthy-broiler isolates $(0 / 20 ; P<0.0001)$. Moreover, tpeL (12/50; 19.3\%; $P=0.0332), p f o A$ (59/62; 95.1\%; $P=0.0017)$ and $c p b 2(49 / 62 ; 79.0 \% ; P<0.0001)$ were all shown to be enriched in NE-linked isolates. Most isolates in lineage $\mathrm{Vf}$ encoded these 4 toxin genes including netB (12/14; 85.7\%), tpeL (10/14; 71.4\%), pfoA $(14 / 14 ; 100 \%)$ and $c p b 2(14 / 14 ; 100 \%)$, supporting the hypothesis of a hypervirulent clone.

Genome-wide association analysis highlighted additional factors that may correlate with widespread nature of lineage $\mathrm{Vf}$ isolates. Aside from the associations of toxin genes tpeL (sensitivity: 64.2\%; specificity: $97.3 \%$; $9 / 14$ isolates) and $n e t B$ (sensitivity: $85.7 \%$; specificity: $72.9 \%$; $12 / 14$ isolates) as described above, collagen adhesin cnaA (sensitivity: $100 \%$; specificity: $85.1 \% ; 14 / 14$ isolates) and a pilinassociated gene (group_5443; sensitivity: 100\%; specificity: $86.5 \%$; all $14 / 14$ isolates) were specifically associated with this lineage of isolates (Additional file 1: Table S10). When we further compared the representative pilin-associated gene group_5443 using NCBI non-redundant (nr) nucleotide database via BLASTn, this gene was detected in both reference chicken isolates EHE-NE18 and Del1 complete genomes at $100 \%$ identity, which was suggested as a hypothetical protein in the annotated file (Additional file 1: Table S10). Other lineage Vf-associated genes including
ABC transporter-related genes ( $n=4$; group_1636, group_ 3194, group_2785, group_3195; sensitivity: 100\%; specificity: $82.4 \%$ ) and phage-associated genes including capsid protein (group_1646), phage-regulatory protein (group_ 6371) and endolysin (group_4126) were also identified.

Adhesin is an important virulence factor in broilerlinked NE [14, 18], and in this study we found that adhesin genes (at least one variant) were overall enriched $(P<0.0001)$ in NE-linked isolates $(58 / 62 ; 93.5 \%)$ vs healthy isolates $(9 / 20 ; 45 \%)$. Among all related adhesin variants, cna, cnaA and cnaD genes were significantly enriched in NE-associated $C$. perfringens isolates $(P<0.05)$, linking these genes to NE-disease development (Additional file 2: Figure S6). Importantly, environmental isolates encoded comparable virulence gene profiles, suggesting potential reservoir including soil and feeds. Indeed, environmental isolate $C$. perfringens FR063 was found to encode the NE-linked netB.

Previous studies $[20,23]$ have indicated that acquired AMR genes are not widespread, and a total of 7 AMR genes were detected across 88 isolates (Fig. 3). Tetracycline resistance genes tet $A(P)$ and tet $B(P)$ were encoded in the greatest number of genomes (44 and 32 isolates respectively), with erythromycin-resistance genes $\mathrm{ermB}$ and erm $Q$ encoded in 2 and 4 isolates respectively. Macrolide-resistant efflux-pump gene mefA was detected in two sub-lineage $\mathrm{Vf}$ isolates, while multidrug-resistant gene emeA was detected in one isolate SYD-NE41 [24]. Notably, approximately half $(47.7 \%)$ of healthy-broiler and NE-linked isolates $(n=42)$ did not carry any acquired AMR genes.

The presence of plasmid(s) was predicted in all genomes using a reference-based sequence-search approach. Overall, 43 out of $88(\sim 48.8 \%)$ isolates carried at least 1 plasmid (18 isolates carried 1 plasmid, 15 isolates harboured 2 plasmids, 4 isolates harboured 3 plasmids, 6 isolates carried 4 plasmids; detailed in Additional file 1: Table S11). Geographical association analysis indicated that two specific plasmids were present in birds from Europe, Australia and North America - plasmids pCP15_1 and pCP15_2 which were first identified in isolate CP15 from an NE-linked chicken in USA (Additional file 2: Figure S7). These two reference plasmids did not carry any well-studied virulence-related genes, nevertheless, the re-annotation of plasmid genes using genus-specific database indicated that this small 14-kb plasmid pCP15_2 encoded a number of genes associated with sugar metabolism including phosphotransferase system (PTS), and sugar transporters sub-units $(n=5$; Additional file 1: Table S12). In terms of plasmid types, European isolates carried 13 different types of isolates, Australian 5 types and USA 6 types. Australian isolates were not found to encode a 'continent-specific', plasmid type, while Europe had 9 unique plasmid types. Plasmids 


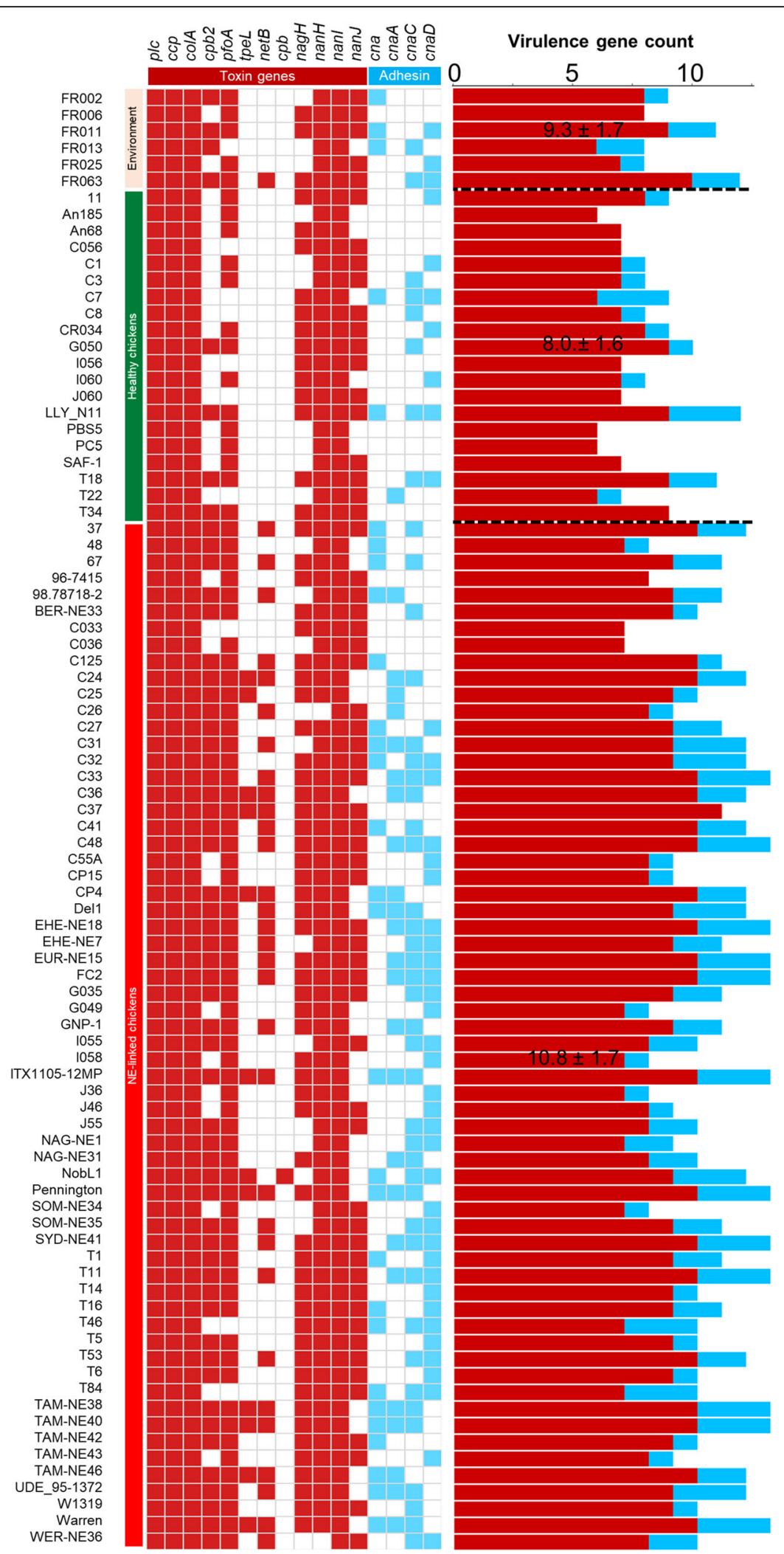

Fig. 4 Virulence profiles of toxin genes and collagen adhesin genes categorised by host health states of bacterial isolates. Coloured cells indicate presence of gene. Data: Mean \pm S.D. in virulence gene count include toxin (red) and adhesin (light blue) genes 
pDel1_4 and pCW3 were the common plasmids detected in isolates from England, Finland and Denmark (Additional file 2: Figure S8). Importantly, both plasmids pDel1_4 (Additional file 1: Table S13) and pCW3 (Additional file 1: Table S14) belonged to conjugative plasmid pCW3 family, carrying AMR genes tet $A(P)$ and tet $B(P)$ and adhesin gene cnaC; sharing highly similar genomic characteristics including plasmid size $(47-49 \mathrm{~kb})$ and CDS number (50-55; Additional file 1: Table S15) [25].

\section{Specific microbiota signatures identified in broiler caecal contents}

We next performed a small exploratory/pilot study to profile poultry gut microbiota bacterial members using caecal content samples obtained from 11 individual broilers representing $3 \mathrm{NE}$ birds, 3 healthy birds and 5 sub-clinical NE birds (Fig. 5 a-d).

Principle Component Analysis (PCA) did not indicate distinct clustering of samples; suggesting a lack of distinctive microbiota signatures between diseased and healthy broilers; however healthy caecal samples appeared to have an inverse relationship with Enterococcus (Additional file 2: Figure S9). Notably, disease-specific profiles might be masked by the fact that a probiotic mix was given to these broilers $(n=11)$ as a preventative measure against NE development. Therefore, raw reads from these genera were removed and another PCA was performed to understand the impact of other secondary or low abundance microbiota members. Again, health vs. disease-status clustering was not observed, however secondary NE-associated profiles did appear to positively correlate with genus Clostridium, with LDA analysis also indicating a Clostridium enriched NE caecal microbiome (mean relative abundance: $0.44 \%$ vs $0.03 \%$ in healthy individuals, Fig. 5e).

Diversity analyses (including Inverse Simpson index, Shannon-weaver index and Fisher index) indicated there was no significant difference $(P>0.05$; ANOVA $)$ in genus diversity between groups (Additional file 2: Figure S10). Relative bacterial genus abundance in each caecal sample was constructed to visualise microbiota profiles (Fig. 5). Thirty-seven genera were represented, with Bifidobacterium and Lactobacillus most abundant, which likely reflected the probiotic supplementation in the chicken feed. A number of secondary genera, which are usual intestinal microbiota members, were detected in these samples (relative abundance $<10 \%$ in each sample) including Blautia, Coprococcus, Dorea and Oscillospira. Blautia was more abundant in health-associated caecal microbiomes (mean abundance: $3.06 \pm 2.84 \%$ ) compared to diseased-associated caecal microbiomes NE (0.72 \pm $0.5 \%)$ and SNE $(0.14 \pm 0.89 \%)$. The microbiota member Enterococcus, which is widely used as veterinary probiotic (especially Enterococcus faecium), was found at high levels in broilers NE2 (50.3\%) and SNE5 (31.0\%). Certain genera appeared to be more abundant in disease-linked NE and SNE samples including Enterococcus and Bacteroides (Additional file 2: Figure S11).

An additional paired-end BLASTn approach, to assign species level 16S rRNA sequences, indicated several important genera were present (Additional file 2: Figure S12) [26]. Lactobacillus reuteri (common broiler gut member, also widely used as probiotic supplement), Lactobacillus salivarius (common swine gut microbiota member used as broiler probiotic that improves production and general health) and Lactobacillus vaginalis (frequently found in broiler gut and a persistent gut coloniser) were the main species within the Lactobacillus genus [27-30]. Enterococcus genus primarily consisted of species Enterococcus faecium, a widely-used probiotic reported to promote broiler growth and suppress C. jejuni and $C$. perfringens infections, while stimulating the growth of Lactobacillus and Bifidobacterium [31, 32]. Importantly, Clostridium genus was mainly assigned to C. perfringens sequences, denoting the potential NE-link of $C$. perfringens origins in NE-broilers particularly NE3, where C. perfringens strains C036 and J36 were isolated from the same NE bird.

\section{Discussion}

Clostridium, particularly C. perfringens, is consistently described as the primary infectious agent to cause chicken NE. As $C$. perfringens thrives at ambient bird body temperature (i.e. $40-42{ }^{\circ} \mathrm{C}$ ), with a doubling time $<$ 8 mins in vitro (the shortest generation time known for a microorganism), this may link to its ability to rapidly overgrow and cause disease pathology $[5,33]$. In this study, we profiled the genomes of $C$. perfringens isolates, including newly sequenced strains, across a geographically diverse and varied health status sample collection. Genome-wide analysis revealed positive associations of important toxin genes with broiler-NE, and we identified a globally-disseminated potentially hypervirulent lineage Vf, which comprised isolates encoding important toxin genes netB and tpeL $[13,34]$.

In silico toxin profiling indicated that the NetB toxin, which has been identified as an essential toxin in NE development, $[13,34]$, was present in $\sim 50 \%$ of the NE isolates, with environmental samples also encoding this toxin, which may act as potential reservoirs, linked to NE outbreaks [35]. The fact that netB gene was exclusively encoded in NE-linked broiler isolates, when compared to healthy isolates, further supported the strong association of this toxin and NE pathogenesis.

Other virulence factors have also been implicated in NE pathogenesis. Several studies have indicated that collagen adhesin (encoded by cna) [14, 18, 36, 37] may facilitate bacterial colonisation within the chicken gut. 
a
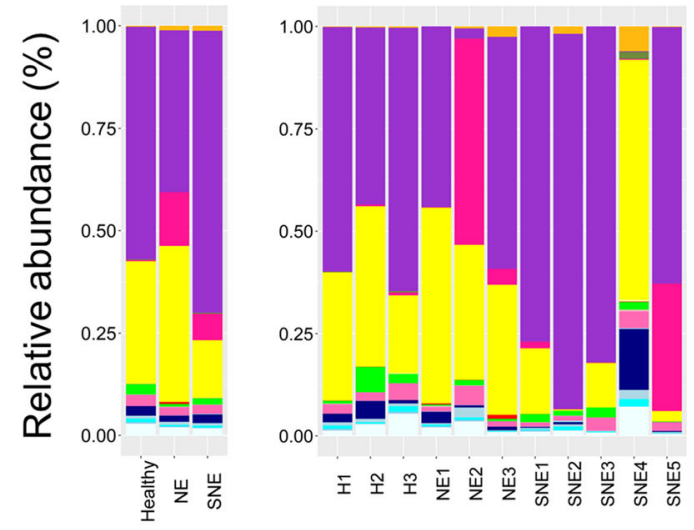

Bacteroides

Bifidobacterium

Staphylococcus

Enterococcus

Lactobacillus

Weissella

Clostridium

Blautia

Coprococcus

Dorea

Anaerotruncus

Faecalibacterium

Oscillospira

Ruminococcus

Coprobacillus

b

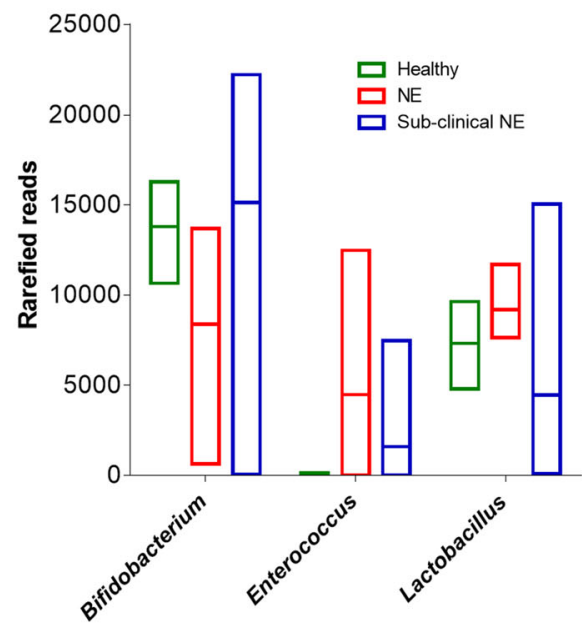

C

Others

d

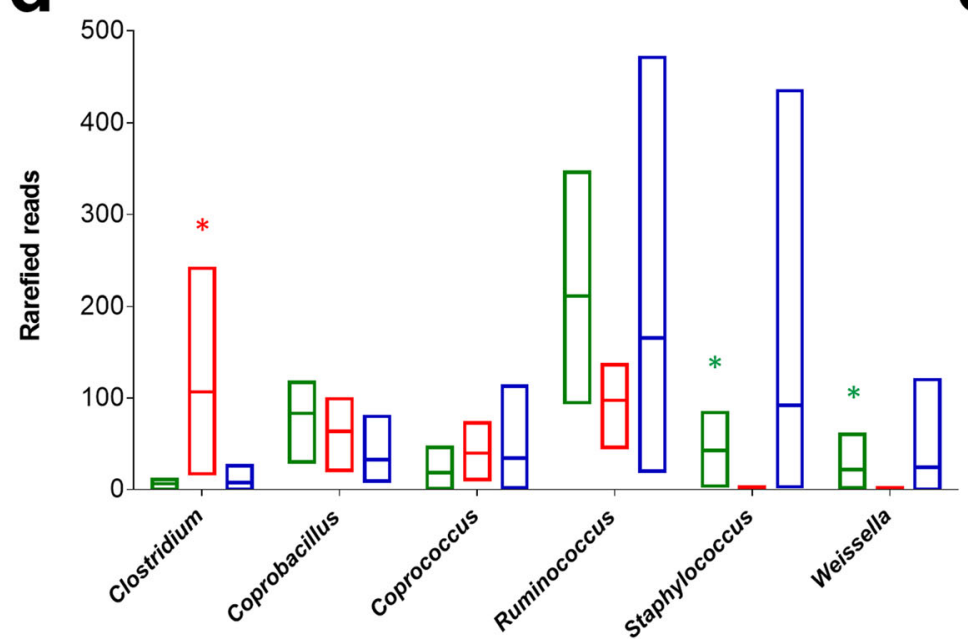

e

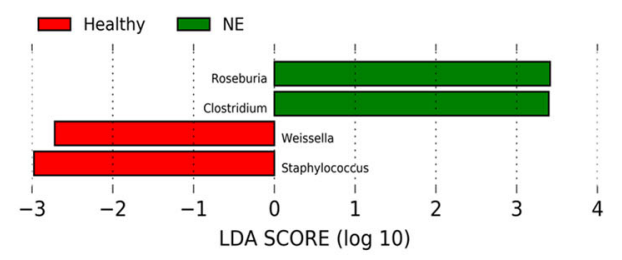

Fig. 5 a Relative abundance of Operational Taxonomical Units (OTUs) based on 16S rRNA amplicon sequencing in 11 individual caecal samples on genus level, with classified comparisons on healthy, NE and SNE (sub-clinical NE) samples. b-d Comparison of taxa reads in 3 groups of samples. e Linear Discriminant Analysis (LDA) indicating significantly enriched taxa 
Our analysis indicated this gene (including its variants cnaA and cnaC) was overabundant in NE-associated isolates when compared to healthy-broiler isolates $(P<0.05)$, which also suggests a positive association with NE outcomes [37].

C. perfringens encodes a diverse array of toxins, and interestingly we also observed that several other accessory toxins were enriched in NE isolates, indicating these may also play an underrated role in broiler NE [38]; PFO, a pore-forming toxin which has been linked with bovine haemorrhagic enteritis [39], and CPB2, or beta2-toxin, another pore-forming cytolytic toxin associated with NE in piglets and enterocolitis in foals [40].

This genomic study indicates a potentially prevalent hypervirulent lineage Vf (comprised 14 isogenic strains; pairwise mean SNPs: 65 in 1810 core genes), with strains obtained from Australia, Canada, Denmark and USA, spanning a period of 20 years (1993-2013). Previous analysis with 9 isolates (out of 14) also indicated these (isogenic) strains grouped within the same lineage [18]. Notably, lineage Vf isolates carried significantly more virulence genes (toxins, including netB and tpeL and collagen adhesin) than isolates in other NE-linked isolates, toxin genes, supports that this lineage may be hypervirulent. TpeL toxin is not typically considered essential for pathogenesis due to its low carriage rate among NElinked C. perfringens isolates (in this study tpeL was exclusively detected in lineage Vf) [41]. Nevertheless, in a broiler-NE infection model, infection with tpeL-positive (also netB-positive) strains induced disease symptoms more rapidly, and with a higher fatality rate, in contrast to tpeL-negative strains encoding only net $B$, highlighting a role for TpeL in more severe chicken-NE pathogenesis [42]. These data also indicate a potential global dissemination of NE-associated virulent genotypes, which is in agreement with a previous study that indicated clonal expansion of $C$. perfringens via multiple-locus variablenumber tandem repeat analysis $(n=328)$ [43]. However, significantly larger sample sizes from various geographical origins will be required for in-depth WGS population structure analysis, if we are to understand the spread of $C$. perfringens in chicken farms worldwide, which will be vital in the context of disease control.

Key C. perfringens virulence factors including toxin and AMR genes are known to be carried on plasmids [44], including the poultry-NE-related toxin netB [13, 34]. The universal $t c p$ conjugative system in majority of plasmids may facilitate horizontal gene transfer and enhance the virulence of $C$. perfringens strains $[45,46]$. As almost half of the genomes carried plasmids $(\sim 48.8 \%)$ this implies widespread plasmid transfer within broilerassociated $C$. perfringens strains. However, as our analysis was carried out using reference-based approaches, in some cases, fragmented short-read sequenced genome assemblies from public databases this may not readily identify plasmid sequences. Indeed, within lineage $\mathrm{Vf}$ we did not observe the expected high carriage rates of plasmids encoding net $B$ [47]. The availability of long-read sequencing (e.g. PacBio and Nanopore) will improve investigations into $C$. perfringens, as plasmids can be sequenced and predicted more accurately despite encoding numerous tandem repeats [48, 49].

In this study, we also analysed C. perfringens isolates obtained from healthy or asymptomatic birds, with several isolates (LLY_N11 and T18) encoding comparable numbers of virulence genes when compared to broilerNE isolates $(n>10)$. Importantly, healthy-broiler isolate LLY_N11 (netB-negative strain, encoded $p f o A$ and $c p b 2$ ) has previously been shown to successfully induce NE in an experimental model $[4,50]$. These data highlight the important role other host factors may play in prevention of overt disease e.g. the chicken gut microbiota. Gutassociated microbial ecosystems are known to play a key colonisation resistance role, preventing overgrowth of so-called pathobionts, or infection by known enteric pathogens (e.g. Salmonella).

In this exploratory/pilot broiler microbiome study, healthy broiler caecal microbiomes appeared to have enhanced abundance of the genera Blautia. Members of the Blautia genus are known to be butyrate producers, and reductions in this genus have previously been associated with a $C$. jejuni infection model [51]. As butyrate is an important energy source for intestinal cells, these Blautia spp. may act as key beneficial microbiota members, serving to enhance intestinal health of chickens by strengthening the epithelial barrier, thus preventing pathogenic microbes successfully colonising and initiating disease. In NE caecal samples we observed appearance of the Clostridium genus, which was significantly enriched, albeit at low reads in NE individuals. Further species-level assignment analysis indicated that most Clostridium sequences mapped to C. perfringens, indicating that even a small proportion (mean relative abundance: $0.44 \%$ ) of $C$. perfringens could potentially be lethal to broiler hosts. Therefore, microbiota profiling may be useful as a potential biomarker for NE-onset, however larger studies, with healthy and diseased birds from different flocks, would be required to verify these findings.

Probiotics, including Bifidobacterium and Lactobacillus, and also Enterococcus, have been frequently used in broiler farming primarily for growth-promotion and prevention of bacterial infections $[29,52,53]$. These taxa of beneficial bacteria have been reflected in caecal microbiome analysis, with predominant OTU proportions been assigned to Bifidobacterium and Lactobacillus across all samples. A previous study identified specific antibacterial peptides produced by Bifidobacterium 
longum that may correlate with the proposed probiotic/ pathogen-inhibitory effect against $C$. perfringens [54]. However, several birds did present with SNE and NE suggesting the strains supplemented to these birds may not be effective in reducing the disease burden associated with $C$. perfringens. Further large scale-controlled supplementation trials are required to provide robust evidence for health promotion using probiotics in poultry.

\section{Conclusions}

In conclusion, genomic analysis of 88 broiler-associated C. perfringens isolates indicates positive correlations relating to virulence genes including netB, $p f o, c p b 2$, tpeL and cna variants linked to NE-linked isolates. Furthermore, potential global dissemination of hypervirulent lineage Vf $C$. perfringens strains highlights the need for further investigations, which will require a large worldwide dataset on NE-related $C$. perfringens isolates.

\section{Methods}

\section{Sample collection and bacterial isolation}

Birds (Ross 308 broilers; culled as part of routine farm surveillance) were collected from four external sites (Oxford, UK) reporting both healthy flocks and flocks that had been diagnosed with NE (Additional file 1: Table S8). All samples were collected within $36 \mathrm{~h}$ after culling. All birds were vaccinated using PARACOX ${ }^{\circ}-5$ (MSD, UK), and prior to euthanisation were aged between 19 to 25 days of age. Culled birds were necropsied and putative disease identification performed, followed by caecum content collection. A representative sub-set of caecal content samples were selected and processed to be sequenced (16S rRNA amplicon sequencing) as part of the pilot caecal microbiome study. Isolation of $C$. perfringens was carried out by isolating organs and submerging $0.1 \%$ peptone water (Oxoid, UK) in a 1:10 ratio of organ to peptone. Samples were streaked onto egg yolk agar supplemented with cycloserine (Oxoid, UK) and incubated overnight anaerobically at $37^{\circ} \mathrm{C}$ [55]. Single black colonies were re-streaked on brain heart infusion agar (Oxoid, UK) and incubated anaerobically at $37^{\circ} \mathrm{C}$ overnight. Several colonies were collected and subjected to identification of the plc gene by PCR, followed by $16 \mathrm{~S}$ rRNA full-length amplicon sequencing as described previously for species verification $[56,57]$.

\section{Bacterial isolates and DNA sequencing}

We isolated 22 novel C. perfringens strains from broilers and environmental samples from farms in Oxford, UK. Genomic DNA of these bacterial isolates was extracted using phenol-chloroform method as described previously [57]. Details of these isolates are given in Additional file 1: Table S1. Sequencing was performed at the Wellcome
Trust Sanger Institute using Illumina HiSeq 2500 to generate $125 \mathrm{bp}$ paired-end reads. Illumina reads are available in the European Nucleotide Archive under project PRJEB32760.

\section{Genome assembly and annotation}

Broiler-related C. perfringens genome assemblies (RefSeq) and quality-trimmed sequencing reads (SRA) were retrieved from NCBI databases in May 2019 including available metadata $(n=68)$. A total of 22 newly sequenced genomes were assembled in-house. All adapter-trimmed sequencing reads were used as input for MEGAHIT v1.1.1 [58]. Genome assembly was carried out using MEGAHIT options --k-min 27 --k-max 247 (for paired-end reads $2 \times 250 \mathrm{bp}$ ), --k-min 27 --kmax 97 (for paired-end reads $2 \times 125 \mathrm{bp}$ ), --no-mercy (specifically for generic assembly) and --min-contig-len 300. Over-fragmented draft genome assemblies were excluded from further computational analysis if $>500$ contigs $(n=2)$. Assembly statistics were calculated using custom Perl script and all sequences were checked to have ANI > 95\% with respect to type strain ATCC13124 genome (Additional file 1: Table S2). All genomes were annotated using Prokka v1.13 with specific Clostridium genus (35 Clostridium species from NCBI RefSeq annotations) database with parameters --usegenus --mincontiglen 300 (Additional file 1: Table S3).

\section{Phylogenetic analysis, SNP detection, in silico virulence gene and plasmid detection}

Annotated gff files were used as input for Roary v3.12.0 to construct pangenome with option -e $-\mathrm{n}$ to generate a core gene alignment via MAFFT, -s do not split paralogs, -i to define a gene at BLASTp 90\% identity and -y to obtain gene inference [59]. A total of 20,194 single nucleotide variants (315,715 site alignment from 1810 core genes) were called using snp-sites v2.3.3 [60]. We used the 20,194 site-alignment to infer a ML phylogeny using RAxML v8.2.10 with GTR+ nucleotide substitution model at 100 permutations conducted for bootstrap convergence test [61]. The ML tree constructed was with the highest likelihood out of 5 independent runs (option -N 5). Pairwise SNP distances were calculated using snp-dists v0.2 [62]. ANI was computed using module pyani v0.2.7 [63]. R package rhierBAPS was used for phylogenetic clustering analysis to identify population structure [64].

Nucleotide sequence search was performed using ABRicate v0.8.11 on genome assemblies with coverage $\geq 90 \%$ and sequence identity $\geq 90 \%$ [65]. Toxin database was constructed as previously described [20] and collagen adhesin genes was detailed in Additional file 1: Table S4. Plasmids were predicted computationally using PlasmidSeeker v1.0 where sequence reads are available 
(k-mer coverage $>80 \%$ ), and ABRicate on all genome assemblies, with best-hit approach at query coverage threshold $\geq 70 \%$ and nucleotide identity $\geq 90 \%$ via custom database as detailed in Additional file 1: Table S5.

\section{Genome-wide gene association analysis}

Scoary v1.6.11 was run to draw gene associations at default parameters [66]. Specificity cutoff was set at $80 \%$, sensitivity at $100 \%$ to obtain 63 genes specifically associated with sub-lineage $\mathrm{Vf}$ isolates (Additional file 1: Table S6).

\section{$16 \mathrm{~S}$ rRNA amplicon sequencing and analysis}

Genomic DNA extraction of caecal samples was performed with FastDNA Spin Kit for Soil following manufacturer's instructions and extending the bead-beating step to $3 \mathrm{~min}$ as described previously [26]. Extracted DNA was quantified and normalised to $5 \mathrm{ng} / \mu \mathrm{l}$ for all samples before subject to $16 \mathrm{~S}$ rRNA Illumina MiSeq sequencing library preparation, amplifying V1 + V2 regions of the 16S rRNA gene as detailed in Additional file 1: Table S7 for the primer sequences. PCR amplification conditions were: 1 cycle of $94^{\circ} \mathrm{C}$ for $3 \mathrm{~min}$, followed by 25 cycles of $94{ }^{\circ} \mathrm{C}$ for $45 \mathrm{~s}, 55^{\circ} \mathrm{C}$ for $15 \mathrm{~s}$ and $72{ }^{\circ} \mathrm{C}$ for $30 \mathrm{~s}$. Libraries were sequenced on the Illumina MiSeq platform using a read length up to $2 \times 300 \mathrm{bp}$.

Sequencing reads were analysed using OTU clustering methods via QIIME v1.9.1 using SILVA_132 as reference database to assign OTU by clustering at $97 \%$ similarity $[67,68]$. Briefly, paired-end sequences were merged using PEAR, followed by quality filtering using split_libraries_fastq.py, chimera identification using identify_ chimeric_seqs.py and chimera removal using filter_fasta.py [69]. OTU picking step was run using open reference approach pick_open_reference_otus.py which does not discard unassigned reads in the final output. BIOM output file was visualised on MEGAN6 [70]. Paired-end approach of taxa assignment using BLASTn as described previously [26].

Caecal contents were processed to generate an average of 116,967 (range: 80426-152,763) raw sequence reads per sample, with an average of 606 (range: 364-1559) OTUs assigned in each sample, clustering at $97 \%$ similarity (Additional file 1: Table S8). Rarefaction analysis supports the availability of sufficient sequence reads to achieve asymptotic based on rarefied reads (normalized to the lowest reads of all samples), i.e. optimal diversity of richness (range: 15-23 genera, 15-25 families) in each individual sample to represent each member of the microbiota (Additional file 2: Figure S1).

LDA was performed using LEfSe via Galaxy server to identify significantly enriched taxa in the dataset. Alpha value for non-parametric Kruskal-Wallis test was set at 0.05 and threshold on LDA score at 2.0 for statistical significance. Graph was illustrated using the LEfSe plotting module [71].

$\mathrm{R}$ package vegan function rarecurve was used to draw rarefaction curves using rarefied reads (normalised to the lowest-read sample as implemented in MEGAN6). Diversity indices including Inverse Simpson index, Shannon index and Fisher index were computed using $\mathrm{R}$ package vegan function diversity [72].

\section{Statistical analysis and graphing}

Venni 2.1 was used to analyse plasmid data [73]. Graphpad PRISM v6.0 was used for various statistical analyses, $\mathrm{R}$ package ggplot 2 was used for various plotting.

\section{Supplementary information}

Supplementary information accompanies this paper at https://doi.org/10. 1186/s42523-019-0015-1.

Additional file 1. Supplementary tables.

Additional file 2. Supplementary figures.

\section{Abbreviations}

ANI: Average Nucleotide Identity; LDA: Linear Discriminant Analysis; ML: Maximum-likelihood; NE: Necrotic enteritis; PCA: Principal Component Analysis; PFO: Perfringolysin O; SNE: Sub-clinical Necrotic Enteritis; WGS: Whole Genome Sequencing

\section{Acknowledgments}

This research was supported in part by the NBI Computing infrastructure for Science (CiS) group through the provision of a High-Performance Computing (HPC) Cluster. We also thank the sequencing team at Wellcome Trust Sanger Institute for genome sequencing.

Ethics approval and consent to participation

Not applicable.

\section{Authors' contributions}

RK and LJH designed the study. RK processed the sequencing data, performed the genomic and $16 \mathrm{~S}$ rRNA amplicon analysis, and graphed the figures. SC provided essential assistance in genome assembly and genomic analysis. RK and LJH analysed the data and co-wrote the manuscript along with JB, RAD and GD. CL processed the caecum content samples and sequencing library. RK performed the full-length $16 \mathrm{~S}$ rRNA PCR. JB collected the broiler faecal and caecal samples, isolated bacterial strains. RK and HB extracted genomic DNA from pure cultures for genome sequencing, which was supported by DP. All authors read and approved the final manuscript.

\section{Funding}

This work was supported by a Wellcome Trust Investigator Award (100974/C/ 13/Z), and the Biotechnology and Biological Sciences Research Council (BBSRC); Institute Strategic Programme Gut Microbes and Health BB/ R012490/1, and its constituent project(s) BBS/E/F/000PR10353 and BBS/E/F/ 000PR10356, and Institute Strategic Programme Gut Health and Food Safety BB/J004529/1 to LJH. JB and RAD were supported by Arden Biotechnology Ltd., Boole Technology Centre, Lincoln. The funders had no role in study design, data collection and interpretation, or decision to submit this work for publication.

\section{Availability of data and materials}

The key datasets analysed during the current study are available as follows: 1. Sequence reads for 22 newly-sequenced Clostridium perfringens strains were deposited under project accession number PRJEB32760.

2. $16 \mathrm{~S}$ rRNA sequence reads of 11 caecal samples were deposited under project accession number PRJEB33036. 
3. Phylogenetic tree aligned with metadata and virulence profiles is available in iTOL: https://itol.embl.de/tree/149155196252227531559222489

\section{Consent for publication}

Not applicable.

\section{Competing interests}

The authors declare that they have no competing interests.

\section{Author details}

${ }^{1}$ Gut Microbes and Health, Quadram Institute Bioscience, Norwich, UK. ${ }^{2}$ University of Lincoln, Lincoln, UK. ${ }^{3}$ Department of Medicine, University of Cambridge, Cambridge, UK.

\section{Received: 8 July 2019 Accepted: 23 September 2019}

Published online: 18 October 2019

\section{References}

1. About chickens [https://www.ciwf.org.uk/farm-animals/chickens/]. Accessed 9 May 2019

2. Keyburn AL, Boyce JD, Vaz P, Bannam TL, Ford ME, Parker D, Di Rubbo A Rood JI, Moore RJ. NetB, a new toxin that is associated with avian necrotic enteritis caused by Clostridium perfringens. PLoS Pathog. 2008:4(2):e26.

3. Lepp D, Roxas B, Parreira VR, Marri PR, Rosey EL, Gong J, Songer JG, Vedantam G, Prescott JF. Identification of novel pathogenicity loci in Clostridium perfringens strains that cause avian necrotic enteritis. PLoS One. 2010;5(5):e10795.

4. Li C, Yan X, Lillehoj HS. Complete Genome Sequence of Clostridium perfringens LLY N11, a Necrotic Enteritis-Inducing Strain Isolated from a Healthy Chicken Intestine. Genome Announc. 2017;5(44):e01225-17.

5. Kiu R, Hall LJ. An update on the human and animal enteric pathogen Clostridium perfringens. Emerg Microb Infect. 2018;7(1):141.

6. Timbermont L, Haesebrouck F, Ducatelle R, Van Immerseel F. Necrotic enteritis in broilers: an updated review on the pathogenesis. Avian Pathol. 2011;40(4):341-7.

7. Olkowski AA, Wojnarowicz C, Chirino-Trejo M, Laarveld B, Sawicki G. Subclinical necrotic enteritis in broiler chickens: novel etiological consideration based on ultra-structural and molecular changes in the intestinal tissue. Res Vet Sci. 2008:85(3):543-53.

8. Drew MD, Syed NA, Goldade BG, Laarveld B, Van Kessel AG. Effects of dietary protein source and level on intestinal populations of Clostridium perfringens in broiler chickens. Poult Sci. 2004;83(3):414-20.

9. Stanley D, Wu SB, Rodgers N, Swick RA, Moore RJ. Differential responses of cecal microbiota to fishmeal, Eimeria and Clostridium perfringens in a necrotic enteritis challenge model in chickens. PLoS One. 2014:9(8):e104739.

10. Yang WY, Lee $Y, \mathrm{Lu} \mathrm{H}$, Chou CH, Wang C. Analysis of gut microbiota and the effect of lauric acid against necrotic enteritis in Clostridium perfringens and Eimeria side-by-side challenge model. PLoS One. 2019;14(5):e0205784.

11. Palliyeguru MWCD, Rose SP. Sub-clinical necrotic enteritis: its aetiology and predisposing factors in commercial broiler production. Worlds Poultry Sci J. 2014;70(4):803-15.

12. Abildgaard L, Sondergaard TE, Engberg RM, Schramm A, Hojberg O. In vitro production of necrotic enteritis toxin $B, N e t B$, by netB-positive and netBnegative Clostridium perfringens originating from healthy and diseased broiler chickens. Vet Microbiol. 2010;144(1-2):231-5.

13. Rood JI, Keyburn AL, Moore RJ. NetB and necrotic enteritis: the hole movable story. Avian Pathol. 2016;45(3):295-301.

14. Ronco $\mathrm{T}$, Stegger $\mathrm{M}, \mathrm{Ng} \mathrm{KL}$, Lilje $\mathrm{B}$, Lyhs U, Andersen PS, Pedersen K. Genome analysis of Clostridium perfringens isolates from healthy and necrotic enteritis infected chickens and turkeys. BMC Res Notes. 2017; 10(1):270.

15. Stanley D, Keyburn AL, Denman SE, Moore RJ. Changes in the caecal microflora of chickens following Clostridium perfringens challenge to induce necrotic enteritis. Vet Microbiol. 2012;159(1-2):155-62.

16. Awad WA, Mann E, Dzieciol M, Hess C, Schmitz-Esser S, Wagner M, Hess M. Age-related differences in the luminal and mucosa-associated gut microbiome of broiler chickens and shifts associated with Campylobacter jejuni infection. Front Cell Infect Microbiol. 2016;6:154.

17. Moore RJ. Necrotic enteritis predisposing factors in broiler chickens. Avian Pathol. 2016:45(3):275-81.
18. Lacey JA, Allnutt TR, Vezina B, Van TTH, Stent T, Han X, Rood Jl, Wade B, Keyburn AL, Seemann T, et al. Whole genome analysis reveals the diversity and evolutionary relationships between necrotic enteritis-causing strains of Clostridium perfringens. BMC Genomics. 2018;19(1):379.

19. Li C, Yan X, Lillehoj HS. Complete genome sequences of Clostridium perfringens Del1 strain isolated from chickens affected by necrotic enteritis. Gut Pathog. 2017;9:69.

20. Kiu R, Caim S, Alexander S, Pachori P, Hall LJ. Probing genomic aspects of the multi-host pathogen Clostridium perfringens reveals significant Pangenome diversity, and a diverse Array of virulence factors. Front Microbiol. 2017:8:2485

21. Chalmers G, Martin SW, Hunter DB, Prescott JF, Weber LJ, Boerlin P. Genetic diversity of Clostridium perfringens isolated from healthy broiler chickens at a commercial farm. Vet Microbiol. 2008;127(1-2):116-27.

22. Nauerby B, Pedersen K, Madsen M. Analysis by pulsed-field gel electrophoresis of the genetic diversity among Clostridium perfringens isolates from chickens. Vet Microbiol. 2003:94(3):257-66.

23. Gholamiandehkordi A, Eeckhaut V, Lanckriet A, Timbermont L, Bjerrum L, Ducatelle R, Haesebrouck F, Van Immerseel F. Antimicrobial resistance in Clostridium perfringens isolates from broilers in Belgium. Vet Res Commun. 2009:33(8):1031-7.

24. Jia B, Raphenya AR, Alcock B, Waglechner N, Guo P, Tsang KK, Lago BA, Dave BM, Pereira S, Sharma AN, et al. CARD 2017: expansion and modelcentric curation of the comprehensive antibiotic resistance database. Nucleic Acids Res. 2017;45(D1):D566-73.

25. Watts TD, Vidor CJ, Awad MM, Lyras D, Rood JI, Adams V. pCP13, a representative of a new family of conjugative toxin plasmids in Clostridium perfringens. Plasmid. 2019;102:37-45.

26. Alcon-Giner C, Caim S, Mitra S, Ketskemety J, Wegmann U, Wain J, Belteki G, Clarke P. Hall LJ. Optimisation of $16 \mathrm{~S}$ rRNA gut microbiota profiling of extremely low birth weight infants. BMC Genomics. 2017;18(1):841.

27. Nakphaichit M, Thanomwongwattana S, Phraephaisarn C, Sakamoto N, Keawsompong S, Nakayama J, Nitisinprasert S. The effect of including Lactobacillus reuteri KUB-AC5 during post-hatch feeding on the growth and ileum microbiota of broiler chickens. Poult Sci. 2011; 90(12):2753-65.

28. Tinrat S, Saraya S, Traidej Chomnawang M. Isolation and characterization of Lactobacillus salivarius MTC 1026 as a potential probiotic. J Gen Appl Microbiol. 2011;57(6):365-78.

29. Messaoudi S, Madi A, Prevost H, Feuilloley M, Manai M, Dousset X, Connil N. In vitro evaluation of the probiotic potential of Lactobacillus salivarius SMXD51. Anaerobe. 2012;18(6):584-9.

30. Wang L, Lilburn M, Yu Z. Intestinal microbiota of broiler chickens as affected by litter management regimens. Front Microbiol. 2016;7:593.

31. Cao GT, Zeng XF, Chen AG, Zhou L, Zhang L, Xiao YP, Yang CM. Effects of a probiotic, Enterococcus faecium, on growth performance, intestinal morphology, immune response, and cecal microflora in broiler chickens challenged with Escherichia coli K88. Poult Sci. 2013;92(11):2949-55.

32. Svetoch EA, Eruslanov BV, Perelygin W, Mitsevich EV, Mitsevich IP, Borzenkov VN, Levchuk VP, Svetoch OE, Kovalev YN, Stepanshin YG, et al. Diverse antimicrobial killing by Enterococcus faecium E 50-52 bacteriocin. J Agric Food Chem. 2008;56(6):1942-8.

33. Li J, McClane BA. Further comparison of temperature effects on growth and survival of Clostridium perfringens type a isolates carrying a chromosomal or plasmid-borne enterotoxin gene. Appl Environ Microbiol. 2006;72(7):4561-8.

34. Keyburn AL, Bannam TL, Moore RJ, Rood Jl. NetB, a pore-forming toxin from necrotic enteritis strains of Clostridium perfringens. Toxins (Basel). 2010;2(7):1913-27.

35. Torok VA, Hughes RJ, Ophel-Keller K, Ali M, Macalpine R. Influence of different litter materials on cecal microbiota colonization in broiler chickens. Poult Sci. 2009;88(12):2474-81.

36. Wade B, Keyburn AL, Haring V, Ford M, Rood Jl, Moore RJ. The adherent abilities of Clostridium perfringens strains are critical for the pathogenesis of avian necrotic enteritis. Vet Microbiol. 2016:197:53-61.

37. Wade B, Keyburn AL, Seemann T, Rood II, Moore RJ. Binding of Clostridium perfringens to collagen correlates with the ability to cause necrotic enteritis in chickens. Vet Microbiol. 2015;180(3-4):299-303.

38. Fisher DJ, Miyamoto K, Harrison B, Akimoto S, Sarker MR, McClane BA. Association of beta2 toxin production with Clostridium perfringens type a human gastrointestinal disease isolates carrying a plasmid enterotoxin gene. Mol Microbiol. 2005;56(3):747-62. 
39. Verherstraeten $S$, Goossens E, Valgaeren B, Pardon B, Timbermont L, Vermeulen K, Schauvliege S, GHaesebrouck F, Ducatelle R, Deprez P. The synergistics necrohemorrhagic action of Clostridium perfringens perfringolysin and alpha toxin in the bovine intestine and against bovine endothelial cells. Vet Res. 2013;44:45.

40. Gibert M, Jolivet-Reynaud C, Popoff MR. Beta2 toxin, a novel toxin produced by Clostridium perfringens. Gene. 1997;203(1):65-73.

41. Bailey MA, Macklin KS, Krehling JT. Low prevalence of netB and tpeL in historical Clostridium perfringens isolates from broiler farms in Alabama. Avian Dis. 2015;59(1):46-51

42. Coursodon CF, Glock RD, Moore KL, Cooper KK, Songer JG. TpeL-producing strains of Clostridium perfringens type a are highly virulent for broiler chicks. Anaerobe. 2012;18(1):117-21.

43. Sawires YS, Songer JG. Clostridium perfringens: insight into virulence evolution and population structure. Anaerobe. 2006:12(1):23-43.

44. Freedman JC, Theoret JR, Wisniewski JA, Uzal FA, Rood Jl, McClane BA. Clostridium perfringens type A-E toxin plasmids. Res Microbiol. 2015;166(4): 264-79

45. Park M, Deck J, Foley SL, Nayak R, Songer JG, Seibel JR, Khan SA, Rooney AP, Hecht DW, Rafii F. Diversity of Clostridium perfringens isolates from various sources and prevalence of conjugative plasmids. Anaerobe. 2015;38:25-35

46. Wisniewski JA, Rood Jl. The Tcp conjugation system of Clostridium perfringens. Plasmid. 2017;91:28-36.

47. Arredondo-Alonso S, Willems RJ, van Schaik W, Schurch AC. On the (im) possibility of reconstructing plasmids from whole-genome short-read sequencing data. Microb Genom. 2017;3(10):e000128.

48. Gonzalez-Escalona N, Allard MA, Brown EW, Sharma S, Hoffmann M. Nanopore sequencing for fast determination of plasmids, phages, virulence markers, and antimicrobial resistance genes in Shiga toxin-producing Escherichia coli. bioRxiv; 2019.

49. Margos G, Hepner S, Mang C, Marosevic D, Reynolds SE, Krebs S, Sing A, Derdakova M, Reiter MA, Fingerle V. Lost in plasmids: next generation sequencing and the complex genome of the tick-borne pathogen Borrelia burgdorferi. BMC Genomics. 2017;18(1):422.

50. Li C, Lillehoj HS, Gadde UD, Ritter D, Oh S. Characterization of Clostridium perfringens strains isolated from healthy and necrotic enteritis-afflicted broiler chickens. Avian Dis. 2017;61(2):178-85.

51. Thibodeau A, Fravalo P, Yergeau E, Arsenault J, Lahaye L, Letellier A. Chicken Caecal microbiome modifications induced by Campylobacter jejuni colonization and by a non-antibiotic feed additive. PLoS One. 2015;10(7): e0131978.

52. Mountzouris KC, Tsirtsikos P, Kalamara E, Nitsch S, Schatzmayr G, Fegeros K. Evaluation of the efficacy of a probiotic containing Lactobacillus, Bifidobacterium, Enterococcus, and Pediococcus strains in promoting broiler performance and modulating cecal microflora composition and metabolic activities. Poult Sci. 2007;86(2):309-17.

53. Gervasi T, Curto RL, Minniti E, Narbad A, Mayer MJ. Application of Lactobacillus johnsonii expressing phage endolysin for control of Clostridium perfringens. Lett Appl Microbiol. 2014:59:355-61.

54. Lee JH, Li X, O'Sullivan DJ. Transcription analysis of a lantibiotic gene cluster from Bifidobacterium longum DJO10A. Appl Environ Microbiol. 2011;77(17): 5879-87.

55. Kotsanas D, Carson JA, Awad MM, Lyras D, Rood JI, Jenkin GA, Stuart RL, Korman TM. Novel use of tryptose sulfite cycloserine egg yolk agar for isolation of Clostridium perfringens during an outbreak of necrotizing enterocolitis in a neonatal unit. J Clin Microbiol. 2010;48(11):4263-5.

56. van Asten AJ, van der Wiel CW, Nikolaou G, Houwers DJ, Grone A. A multiplex PCR for toxin typing of Clostridium perfringens isolates. Vet Microbiol. 2009;136(3-4):411-2.

57. Kiu R, Caim S, Alcon-Giner C, Belteki G, Clarke P, Pickard D, Dougan G, Hall ᄂ. Preterm infant-associated Clostridium tertium, Clostridium cadaveris, and Clostridium paraputrificum strains: genomic and evolutionary insights. Genome Biol Evol. 2017:9(10):2707-14.

58. Li D, Liu CM, Luo R, Sadakane K, Lam TW. MEGAHIT: an ultra-fast singlenode solution for large and complex metagenomics assembly via succinct de Bruijn graph. Bioinformatics. 2015:31(10):1674-6.

59. Page AJ, Cummins CA, Hunt M, Wong VK, Reuter S, Holden MT, Fookes M, Falush D, Keane JA, Parkhill J. Roary: rapid large-scale prokaryote pan genome analysis. Bioinformatics. 2015;31(22):3691-3.
60. Page AJ, Taylor B, Delaney AJ, Soares J, Seemann T, Keane JA, Harris SR. SNP-sites: rapid efficient extraction of SNPs from multi-FASTA alignments. Microb Genom. 2016;2(4):e000056.

61. Stamatakis A. RAxML version 8: a tool for phylogenetic analysis and postanalysis of large phylogenies. Bioinformatics. 2014;30(9):1312-3.

62. Seemann T, Klotzl F, Page AJ: snp-dists. In., 0.2 edn; 2018: Convert a FASTA alignment to SNP distance matrix.

63. Pritchard L, Glover RH, Humphris S, Elphinstone JG, Toth IK. Genomics and taxonomy in diagnostics for food security: soft-rotting enterobacterial plant pathogens. Anal Methods. 2016;8(1):12-24.

64. Tonkin-Hill G, John AL, Stephen DB, Simon DWF, Jukka C. RhierBAPS: An R Implementation of the Population Clustering Algorithm hierBAPS. Wellcome Open Research. 2018;3(July):93.

65. Seemann T: ABRicate. In., 0.5 edn; 2018: Mass screening of contigs for antimicrobial resistance or virulence genes.

66. Brynildsrud O, Bohlin J, Scheffer L, Eldholm V. Rapid scoring of genes in microbial pan-genome-wide association studies with Scoary. Genome Biol. 2016;17:238

67. Caporaso JG, Kuczynski J, Stombaugh J, Bittinger K, Bushman FD, Costello EK, Fierer N, Pena AG, Goodrich JK, Gordon Jl, et al. QIIME allows analysis of high-throughput community sequencing data. Nat Methods. 2010:7(5):335-6.

68. Quast C, Pruesse E, Yilmaz P, Gerken J, Schweer T, Yarza P, Peplies J, Glockner FO. The SILVA ribosomal RNA gene database project: improved data processing and web-based tools. Nucleic Acids Res. 2013;41(Database issue):D590-6.

69. Zhang J, Kobert K, Flouri T, Stamatakis A. PEAR: a fast and accurate Illumina paired-end reAd mergeR. Bioinformatics. 2014;30(5):614-20.

70. Huson DH, Beier S, Flade I, Gorska A, El-Hadidi M, Mitra S, Ruscheweyh HJ, Tappu R. MEGAN Community edition - interactive exploration and analysis of large-scale microbiome sequencing data. PLoS Comput Biol. 2016;12(6): e1004957.

71. Segata N, Izard J, Waldron L, Gevers D, Miropolsky L, Garrett WS, Huttenhower C. Metagenomic biomarker discovery and explanation. Genome Biol. 2011;12(6):R60.

72. R Development Core Team. R: A language and environment for statistical computing. Vienna: R Foundation for Statistical Computing: 2010

73. Venny. An interactive tool for comparing lists with Venn's diagrams. [http:// bioinfogp.cnb.csic.es/tools/venny/index.html]. Accessed 16 May 2019.

\section{Publisher's Note}

Springer Nature remains neutral with regard to jurisdictional claims in published maps and institutional affiliations.

Ready to submit your research? Choose BMC and benefit from:

- fast, convenient online submission

- thorough peer review by experienced researchers in your field

- rapid publication on acceptance

- support for research data, including large and complex data types

- gold Open Access which fosters wider collaboration and increased citations

- maximum visibility for your research: over $100 \mathrm{M}$ website views per year

At BMC, research is always in progress.

Learn more biomedcentral.com/submission 